Original Paper http://ajol.info/index.php/ijbcs http://indexmedicus.afro.who.int

\title{
Mercure: sources d'émission, toxicité, contamination du milieu aquatique et particularité du Benin
}

\author{
Hermione W. DEGILA ${ }^{1,3^{*}}$, N.B. Nadia AZON ${ }^{1,3}$, Julien G. ADOUNKPE ${ }^{2}$, \\ A. V. Onésime AKOWANOU ${ }^{1}$ et Martin P. AÏNA ${ }^{1,3}$ \\ ${ }^{l}$ Laboratoire des Sciences et Techniques de l'Eau de l'Institut National de l'Eau, Université d'Abomey-Calavi, \\ Benin. \\ ${ }^{2}$ Laboratoire d'Hydrologie Appliquée de l'Institut National de l'Eau, Université d'Abomey-Calavi, Benin. \\ ${ }^{3}$ Laboratoire de Surveillance Environnementale du Ministère du Cadre de Vie et du Développement Durable. \\ *Auteur correspondant ; E-mail : hermywolf@gmail.com
}

\section{RESUME}

La prise de conscience collective du pouvoir létal du mercure suite aux intoxications massives au Japon et en Irak ont conduit à l'adoption de la convention de Minamata sur le mercure que le Bénin a signé et ratifié. Le présent article fait la synthèse des connaissances sur son émission, sa toxicité en milieu aquatique avec un accent sur la contamination aquatique au Bénin. Pour y parvenir, des publications scientifiques et les archives des ministères de l'environnement et de la santé au Bénin ont été consultées. Il ressort que, la production artisanale à petite échelle de l'or et la combustion de charbon constituent les principales sources d'émission de mercure dans le monde avec respectivement $37,1 \%$ et $24,2 \%$ des émissions anthropiques. Au Bénin, les piles contenant du mercure (49\%) l'orpaillage artisanal (22\%) et les dépôts informels de déchets généraux (13\%) sont les principales sources potentielles d'émission anthropique. Les régions actuellement les plus émettrices de mercure sont l'Asie et l'Afrique sub-saharienne avec respectivement 50\% et 16,8\% des émissions anthropiques. La formation du méthylmercure en milieu aquatique sous l'influence de divers facteurs et sa bioaccumulation sont principalement responsable des effets toxiques sur l'homme.

(C) 2019 International Formulae Group. All rights reserved.

Mots clés : Environnement, pollution, mercure, méthyl mercure, milieu aquatique, toxicité.

\section{Mercury: sources of emission, toxicity, contamination of aquatic environment and particularity of Benin republic.}

\begin{abstract}
The massive mercury poisoning that has occurred in Japan and Iraq has led to a global awareness of the lethal power of this metal with the adoption by the international community of the Minamata Convention signed and ratified by Benin Republic. This article aims to synthesize knowledge on mercury through its emission sources, its toxicity with a focus on aquatic contamination in Benin. Thus, a review of scientific publications as well as the consultation of the archives of the Ministries of Environment and Health in Benin were carried out. Analyses of the various documents show that artisanal small-scale gold production and coal combustion are the main sources of mercury emission worldwide, with respectively $37.1 \%$ and $24.2 \%$ of
\end{abstract}


anthropogenic emissions. Asia and sub-Saharan Africa would be the largest contributors to anthropogenic emissions with respectively $50 \%$ and $16.8 \%$. In Benin, the main potential sources of anthropogenic emissions are mercury containing batteries (49\%), followed by artisanal gold panning (22\%) and informal deposits of general waste $(13 \%)$. With regard to the behavior of mercury in the aquatic environment and its toxicity, it appears that methyl mercury is mainly responsible for toxic effects.

(C) 2019 International Formulae Group. All rights reserved.

Keywords: Environment, pollution, mercury, methyl mercury, aquatic environment, toxicity Bénin.

\section{INTRODUCTION}

La présence et la dispersion des polluants dans l'environnement sont l'objet d'attentions depuis de nombreuses années. Parmi les contaminants majeurs de l'environnement, les métaux lourds posent de sérieux problèmes écologiques, tant par le caractère ubiquiste de leur présence que par leur toxicité et leur bioaccumulation potentielle dans plusieurs espèces animales, notamment aquatiques, (Katemo et al., 2010). Le mercure, l'un des métaux lourds les plus toxiques, est une substance chimique préoccupante à l'échelle mondiale du fait de sa propagation atmosphérique, et de sa persistance dans l'environnement. Seul métal liquide à température ambiante, il possède une pression de vapeur élevée, ce qui explique sa distribution relativement homogène à l'échelle planétaire. Le mercure émis dans l'atmosphère restera dans le système atmosphère-océanterre pendant environ 3000 ans avant d'être stocké dans les sédiments au fond des océans (Selin et al., 2008). C'est un élément métallique naturellement présent dans la croûte terrestre à une concentration d'environ $0,02 \mathrm{mg} / \mathrm{kg}$. De plus, le mercure, largement utilisé dans diverses activités anthropiques (productions industrielles de batteries, câbles et interrupteurs électriques, appareils de mesure, amalgames dentaires, lampes, production artisanale ou industrielle de l'or, l'incinération des déchets, etc.) est abondamment rejeté dans l'environnement (UNEP, 2013).

Dans l'environnement, il existe sous diverses espèces inorganiques et organiques. Le passage d'une forme à l'autre est contrôlé par une multitude de processus qui comprennent des réactions photochimiques, des réactions d'oxydoréduction, catalysées ou non par des agents bactériens (Thomassin et al., 2003). L'intérêt du monde scientifique et de la communauté internationale pour cet élément résulte principalement de son potentiel de bioaccumulation dans les écosystèmes et sa grande toxicité sur la santé humaine et l'environnement.

Les premières maladies reconnues comme provoquées par le mercure, ont été observées au XIXe siècle chez les chapeliers. Ceux-ci utilisaient des sels de mercure pour travailler les poils d'animaux en vue de la confection de feutres. Les chapeliers inhalant les vapeurs de mercure présentaient des troubles du langage et de la vision, pouvant aller jusqu'à des troubles neurologiques caractérisant l'hydrargie, maladie dégénérative (Marusczak, 2010). Cependant, il a fallu attendre le milieu du XXe siècle avec les intoxications massives survenues au Japon en 1956 et en Irak en 1971 pour une prise de conscience du pouvoir létal du mercure.

Aujourd'hui encore, la toxicité du mercure demeure une préoccupation importante dans de nombreux pays. Par exemple au Japon la consommation de certaines populations de mammifères marins est interdite parce que leurs teneurs en méthyle mercure sont trop élevées (Endo et al., 2003). En 2007, la Norvège a interdit l'utilisation de mercure probablement pour éviter d'accentuer les risques sanitaires et économiques que constitue la présence du sous-marin U-864 chargé de plus de 60 tonnes de mercure dans les fonds marins du pays.

La toxicité de ce métal se manifeste à la fois sur la faune aquatique et sur l'homme. $\mathrm{Au}$ niveau des organismes aquatiques, l'intoxication au mercure se manifeste par des atteintes des systèmes immunitaire et nerveux, des appareils respiratoire et cardio-vasculaire 
ainsi qu'une atteinte des organes reproducteurs (Diez, 2009 ; Morcillo et al., 2017). Le mercure, après son entrée dans l'organisme par voie respiratoire (branchies, peau ou absorption directe au niveau de la muqueuse intestinale) ou digestive par ingestion de proies contaminées, se répand dans tout le corps et est stocké généralement dans le foie, les reins, la chair le cerveau (Diez, 2009.). Sa toxicité dépend non seulement de la teneur en mercure total et de l'espèce chimique mais également de la faculté d'absorption de l'organisme (Morcillo et al., 2017). L'exposition au mercure peut induire une variété d'effets indésirables chez les poissons aux niveaux physiologique, histologique, biochimique, enzymatique et génétique et des différences de sensibilité, liées au sexe peuvent exister pour certaines espèces (Robinson et al., 2011 ; Morcillo et al., 2017).

Pour ce qui est de l'être humain, la toxicité du mercure dépend à la fois de l'espèce chimique et de la voie d'exposition. L'exposition à la forme élémentaire, peut être transcutanée lorsqu'il est liquide (thermomètre cassés) ou respiratoire lorsqu'il est gazeux. Le mercure élémentaire est bien plus toxique par inhalation avec un taux d'absorption compris entre 75 et $85 \%$ tandis que le mercure inorganique est faiblement absorbé par l'organisme humain avec un taux d'absorption compris entre 7 et $15 \%$ (INERIS, 2006). Toutefois, cette dernière forme chimique peut être à la base d'insuffisances rénales et de lésions gastrointestinales. Le méthylmercure (MeHg), la forme la plus toxique du mercure par ingestion, peut causer des dommages neurologiques graves pour l'homme (Diez, 2009 ; Gibb et O'Leary, 2014; Grandjean et al., 2014.) (Voir Tableau 1) C'est pour limiter les risques sanitaires du mercure sur l'homme que l'OMS a défini des critères pour la consommation de poisson, qui est la source principale de mercure et de méthylmercure pour les populations humaines. Les Doses Hebdomadaires Tolérables Provisoires (DHTP) de 1,6 $\mu \mathrm{g}$ de $\mathrm{MeHg} / \mathrm{kg}$ p.c./semaine (soit $0,23 \mu \mathrm{g} / \mathrm{kg}$ p.c./j) et de $5 \mu \mathrm{g}$ de $\mathrm{Hg}-\mathrm{T} / \mathrm{kg}$ p.c./semaine ont été fixées (OMS,2003). La Commission européenne par Règlement (CE) N466/2006 a fixé des teneurs maximales en mercure total de $0,5 \mathrm{mg} / \mathrm{kg}$ de poids frais pour les produits de la pêche, sauf pour certaines espèces (anguille, mulet, brochet, requin, espadon, marlin, thon, par exemple) pour lesquelles la valeur a été fixée à $1 \mathrm{mg} / \mathrm{kg}$ de poids frais. Le Bénin par arrêté 2007 N0362 : MAEP/D-CAS/SGM/DRH/DP/SA du 30 octobre 2007 a adopté les mêmes valeurs que l'Union Européenne pour les teneurs maximales de mercure dans les produit halieutiques.

Cet article présente une synthèse des connaissances sur les sources d'émission du mercure, son comportement dans les écosystèmes aquatiques avec une attention particulière sur sa méthylation responsable de la toxicité par ingestion comparées aux autres espèces chimiques. La particularité des émissions de mercure ainsi que le niveau de contamination du milieu aquatique au Bénin ont aussi été abordés.

\section{MATERIEL ET METHODES}

Le présent article vise à faire une synthèse des connaissances sur les sources d'émission de mercure et les risques environnementaux et sanitaires liés à sa présence dans les écosystèmes aquatiques. Pour y parvenir, une visite a été effectuée au niveau des Ministères en charge de la santé et de l'environnement au Bénin où les points focaux de la convention de Minamata sur le Mercure ont été rencontrés. Cette visite a permis de recueillir les données sur l'inventaire initial du mercure effectué grâce à l'appui financier $\mathrm{du}$ fond mondial pour l'environnement. De plus, les mots clés (mercure, methylmercure, écosystème aquatique, toxicité, intoxication, poisson) ont permis de retrouver des articles dont les plus pertinents ont été retenus sur la base des résumés puis téléchargés et exploités. Des représentations graphiques des données relatives à l'inventaire du mercure au niveau mondial et au Bénin ont été effectuées à l'aide du logiciel Excel version 2013). 
Tableau 1 : Manifestations cliniques de l'intoxication au mercure chez l'homme.

\begin{tabular}{|c|c|c|}
\hline $\begin{array}{l}\text { Organes } \\
\text { atteints }\end{array}$ & $\begin{array}{l}\text { Symptômes/pathologies } \\
\text { développées }\end{array}$ & Références \\
\hline $\begin{array}{l}\text { troubles et } \\
\text { symptômes } \\
\text { neurologiques }\end{array}$ & $\begin{array}{l}\text { Réflexes tendineux profonds; } \\
\text { perturbation de la coordination } \\
\text { motrice des membres inférieurs; } \\
\text { perturbation de l'organisation de la } \\
\text { Vision spatiale avec diminution de } \\
\text { l'attention et de la sensibilité visuelle } \\
\text { au contraste; } \\
\text { diminution de la dextérité manuelle; } \\
\text { Céphalées ; } \\
\text { trouble de sommeil ; } \\
\text { Altération neurologique; } \\
\text { Tremblements; } \\
\text { Retard de développement de l'enfant } \\
\text { et malformation du fœtus. }\end{array}$ & $\begin{array}{l}\text { (Budtz-Jorgensen et al., 2004; } \\
\text { Margos et Clarkson, 2006; Diez, } \\
\text { 2009; Bensefa- Colas et al., 2011; } \\
\text { Tomicic, et al., 2011; Harari et al., } \\
2012 \text {; Gibb et O'Leary. 2014; } \\
\text { Grandjean et al., 2014; Bose- } \\
\text { O'Reilly et al., 2016.) }\end{array}$ \\
\hline $\begin{array}{l}\text { Troubles } \\
\text { rénaux }\end{array}$ & néphrotoxicité & $\begin{array}{l}\text { (Drake et al., 2001; OMS., 2003; } \\
\text { Yard et al., 2012.) }\end{array}$ \\
\hline $\begin{array}{l}\text { Immuno } \\
\text { toxicité }\end{array}$ & troubles auto immunes & $\begin{array}{l}\text { (Gardner et al., } 2010 \text {; Nyland et al., } \\
\text { 2011.) }\end{array}$ \\
\hline $\begin{array}{l}\text { atteinte du } \\
\text { système cardio } \\
\text { vasculaire }\end{array}$ & & (Gardner et al., 2010.) \\
\hline $\begin{array}{l}\text { effet } \\
\text { cancérigène }\end{array}$ & & (Crespo-Lopez et al., 2009.) \\
\hline
\end{tabular}

\section{RESULTATS}

L'exploitation des différents documents a permis d'aboutir à cette synthèse qui porte sur les sources d'émission du mercure à l'échelle mondiale et au Bénin ainsi que sa toxicité en milieu aquatique.

\section{Emissions de mercure}

Le mercure présent dans l'environnement provient de sources naturelles et des activités anthropiques (UNEP, 2008).

\section{Emissions mondiales du mercure}

Pour ce qui est des sources naturelles, elles concernent les émanations de vapeurs de $\mathrm{Hg}$ qui proviennent de la croûte terrestre (volcans et autres failles) constituent la source primaire du mercure (Stella et al., 2017) Tandis que les réémissions des terres et des océans sont une autre source naturelle de mercure dans l'atmosphère (UNEP, 2008). Ces émissions naturelles sont évaluées à 5400 tonnes par Holmes et al. (2010) et 3700 à 5700 tonnes par Mason (2009) et constituent environ 60 à $69 \%$ des émissions atmosphériques. L'environnement marin, qui représente $70 \%$ de la surface du globe, est responsable de 52\% (2682 tonnes/an) des émissions naturelles de mercure alors que l'environnement terrestre qui occupe $30 \%$ de la surface du globe est responsable de $48 \%$ (2525 tonnes/an) des émissions naturelles 
(Mason, 2009). Ainsi, en considérant la charge de mercure émise par unité de surface, les émissions moyennes produites par l'environnement terrestre sont plus importantes que celles produites par l'environnement marin (Mason, 2009).

Quant aux émissions anthropiques, elles sont globalement estimées à environ 2000 tonnes par an (Selin et al., 2008; Holmes et al., 2010). Pour certains auteurs, près de $80 \%$ des émissions anthropiques primaires du $\mathrm{Hg}$ dans l'atmosphère sont des émissions de $\mathrm{Hg}$ gazeux provenant essentiellement des combustibles fossiles, des mines, de la purification des minerais et de l'incinération des déchets solides (Pacyna et al., 2010 ; Stein et al., 2011 ). Stella et al. (2017) estiment que 15\% des émissions anthropiques sont constituées par des apports directs aux sols sous la forme de fertilisants et de fongicides tandis que les effluents industriels ou miniers sont responsables de $5 \%$ de ces émissions. La Figure 1 réalisée avec les données de AMAP (2013) montre que la production artisanale et à petite échelle de l'or et la combustion de charbon constituent les sources majeures d'émission de mercure dans l'atmosphère avec respectivement $37,1 \%$ et $24,2 \%$ des émissions anthropiques suivi de la production de métaux primaires non ferreux $(9,9 \%)$ et celle de ciment $(8,8 \%)$. La production industrielle de l'or vient en cinquième position avec une contribution ne représentant que 5\% des émissions anthropiques de mercure. Pour l'UNEP 2013, les piles usagées, les lampes fluorescentes aux vapeurs de mercure, les thermomètres à mercure, certaines peintures, d'anciens déchets et matériaux industriels constituent des sources diffuses de rejet de mercure dans l'environnement.

\section{Emissions anthropiques du mercure au Bénin}

Le Bénin n'est pas un pays producteur de mercure, mais cette substance y est présente à travers divers produits et secteurs d'activités. Il s'agit principalement des appareils biomédicaux, des batteries et accumulateurs (piles), des lampes fluorescentes, et du secteur de l'orpaillage.
Les piles contenant du mercure, l'orpaillage artisanal, les dépôts informels de déchets généraux et le brûlage des déchets à l'air libre (sur des sites de décharge ou de manière informelle) représentent les sources potentielles majeures d'émission de mercure au Bénin (Figure 3). Elles contribuent respectivement pour $49 \%, 22 \%, 13 \%$ et $10 \%$ des émissions anthropiques. (Direction Générale de l'Environnement et du Climat, 2018).

L'estimation des émissions/ rejets effectifs à la suite de l'utilisation des différentes sources d'apport dans les diverses activités anthropiques au Bénin a révélé que les trois compartiments de l'environnement (eau, air, sols) sont impactés à des degrés divers. Plus de $42.000 \mathrm{~kg}$ de mercure sont rejetés annuellement dans l'environnement dont plus de $16.000 \mathrm{~kg}$ dans l'air, environ $5.000 \mathrm{~kg}$ dans l'eau et plus de $14.000 \mathrm{~kg}$ dans le sol (Direction Générale de l'Environnement et du Climat, 2018).

La Figure 4 réalisée à partir des données du rapport de l'inventaire initial du mercure de 2017 présente la proportion de mercure reçu par les différents compartiments de l'environnement au Bénin. L'air vient en tête avec $49 \%$ des émissions suivi du sol (39\%) et de l'eau (14\%). De façon spécifique, il est à noter que l'extraction artisanale à petite échelle de l'or émet le mercure dans les trois compartiments de l'environnement contrairement au brûlage des déchets n'émettant que dans l'air et les crèmes et savons éclaircissants dont les émissions sont presque exclusivement dédiées au compartiment eau. Concernant les piles contenant $\mathrm{du}$ mercure, leur utilisation s'accompagne d'une émission équitable du mercure dans l'air et dans le sol (Tableau 2).

\section{Mercure en milieu aquatique}

Les océans constituent le plus grand réservoir de mercure avec près de 300000 tonnes de mercure, (Mason et Sheu, 2002). Le mercure se retrouve généralement dans les océans, sous trois formes chimiques. (i) la forme élémentaire gazeuse $\mathrm{Hg} 0$, (ii) la forme 
de mercure divalent ionique, $\mathrm{Hg}$ (II) qui peut former de complexes inorganiques et organiques, (iii) la forme méthylée, comprenant le methyl mercure et le di methyl mercure (Fitzgerald et al., 2007). Les apports de mercure dans les océans se font principalement par dépôts atmosphériques, secs ou humides, et par les cours d'eau (Mason et Sheu, 2002). En milieu marin et en absence de matière organique, les chlorures de mercure sont les plus abondants (Thomassin et al. , 2003).

$\mathrm{Au}$ niveau des eaux douces, les principales sources de mercure, sont également les dépôts atmosphériques (Graydon et al., 2008), mais aussi les eaux de ruissellement via le carbone organique dissous (Grigal, 2002), et les eaux souterraines (Driscoll et al., 2007; Chouti et al., 2010). Une partie du mercure est réémise vers l'atmosphère par processus photochimique (Mason, 2009). Le mercure est également évacué par les eaux de drainage, ou alors stockée dans les sédiments, essentiellement sous forme de $\mathrm{HgS}$. Enfin, une dernière partie de ce mercure sera convertie en mercure organique, principalement le méthylmercure (Grigal, 2002).

Au niveau des sols et sédiments, le mercure pourrait s'adsorber sur oxyhydroxydes de fer ou de manganèse ou encore à la matière organique. Cette faculté fait de ce milieu un réservoir (Choe et al., 2004.) qui peut libérer le mercure dans la colonne d'eau et d'autres compartiments de l'environnement pendant de longues périodes.(Grigal, 2002; PNUE, 2002). Au niveau des sédiments, les composés de mercure divalent peuvent être réduits en $\mathrm{Hg} 0$ via la lumière ou certaines substances humiques ou entrée dans un processus de méthylation/démethylation biotique ou abiotique (Hamelin et al., 2011; Yu et al., 2012). Dans les sédiments anoxiques, le mercure est principalement présent sous forme de sulfure de mercure $\mathrm{HgS}$, préservé de la méthylation (Marusczak, 2010).

\section{Présence du en mercure en du milieu aquatique au Bénin}

Pour ce qui est du cas particulier du Bénin, il faut noter que de nombreuses études ont porté sur la pollution métallique des écosystèmes aquatiques (Edorh et al., 2009 ; Chouti et al., 2010; Chouti et al., 2011; Youssao et al.2011; Aïna et al., 2012 ; Edorh et al., 2012; Hounkpatin et al., 2012; Youssao et al., 2018 ; Adamou et al., 2019). Mais, peu ont abordé la pollution mercurielle. Ces dernières montrent que la présence du mercure dans l'écosystème aquatique béninois résulte principalement de la production artisanale à petite échelle de l'or dans les départements de l'Atacora (Youssao et al., 2018) et de la culture intensive du coton dans les deux départements au nord du pays (Adam et al., 2010; Youssao et al., 2018). Ces activités induisent une contamination mercurielle du fleuve Ouémé, de ses affluents, de la lagune de Porto-Novo ainsi que du lac Nokoué (Chouti et al., 2010 et 2011 ; Youssao et al., 2018). Toutefois, cette pollution provient également de l'eau souterraine au niveau de la lagune de PortoNovo où Chouti et al. (2011) ont identifié une source ponctuelle de pollution de la lagune par le mercure à Bouédomey par les eaux souterraines. Selon ces mêmes auteurs, «les eaux de Bouédomey autrefois bues par la population continuent d'être utilisées pour différents travaux domestiques » constituant ainsi un danger pour les organismes aquatiques qui y vivent et pour la santé humaine. Le mercure est identifié par Chouti et al. (2010) comme «une des causes de la mauvaise qualité des eaux de la lagune et par conséquent explique en partie la mort des poissons souvent constatée par les pêcheurs ».

S'agissant des sédiments, des teneurs allant de $12.4 \mu \mathrm{g} / \mathrm{kg}$ à $40.1 \mu \mathrm{g} / \mathrm{kg}$ ont été trouvés au niveau des sédiments du Mékrou (Youssao et al., 2018). Pour ce qui est de l'eau les concentrations en mercure total vont de 3 à $25 \mu \mathrm{g} / \mathrm{L}$ au niveau de la lagune de Porto-Novo en saison sèche (Chouti et al., 2010) et de de 0,5 à $1,3 \mu \mathrm{g} / \mathrm{L}$ dans les eaux calme et 
transparente du Mékrou. (Youssao et al., 2018) Cependant, des valeurs de $616,9 \mu \mathrm{g} / \mathrm{L}$ de mercure ont été obtenues dans certaines parties à fort courant d'eau toujours au niveau du Mékrou (Youssao et al., 2018). Concernant la faune aquatique du Lac Nokoué, Aïna et al. (2012) ont obtenu une valeur moyenne en mercure total d'environ $0,02 \mu \mathrm{g} / \mathrm{kg}$ de poids sec pour le tilapia Sarotherodon melenotheron et les crevettes Peneaus SPP.

\section{Méthylation du mercure en milieu aquatique}

L'accumulation du methylmercure le long des réseaux trophiques est responsable du risque sanitaire de ce métal pour l'homme par ingestion. Il importe donc de comprendre le processus conduisant à sa formation ainsi que les facteurs qui influencent ledit processus.

\section{Processus de méthylation.}

La production de méthylmercure ne se fait que dans des conditions anoxiques dans les eaux de fond ou dans le sédiment (Thomassin et al., 2003 ; Duran et al., 2008 ; Ranchou-Peyruse et al., 2009). C'est dans les premiers centimètres de la colonne sédimentaire où l'anoxie apparaît généralement que le maximum de méthylation est observé et le processus peut être abiotique et / ou microbiologique. Même si l'importance relative des deux processus est mal connue, il est admis que la méthylation du mercure en milieu aquatique est principalement microbienne (Ullrich et al., 2001).

Le transfert d'un groupe méthyl à partir de composés organiques vers l'ion mercure $\left(\mathrm{Hg}^{2+}\right)$ nécessite un cabanion fortement instable dans l'eau comme la méthylcabalamine $\left(\mathrm{CH}_{3} \mathrm{CoB} 12\right)$, dérivée de la vitamine $\mathrm{B} 12$ selon la réaction chimique suivante (Ullrich et al., 2001).

$$
\begin{aligned}
& \mathrm{CH}_{3} \mathrm{CoB} 12+\mathrm{Hg}^{2+}+\mathrm{H}_{2} \mathrm{O} \longrightarrow \mathrm{CH}_{3} \mathrm{Hg}^{+} \\
& +\mathrm{Hg}^{2+}+\mathrm{H}_{2} \mathrm{OCoB} 12^{+} \\
& \text {La méthylcobalamine est un produit }
\end{aligned}
$$
de synthèse biologique de nombreux micro- organismes rencontrés dans les sédiments oxiques, sub-oxiques et anoxiques. Toutefois c'est dans des conditions anaérobiques que les bactéries sulfate-réductrices (BSR) ont été identifiées comme les principaux agents de la méthylation biotique du mercure inorganique (Hamelin et al., 2011) par l'intermédiaire de ce dérivé vitaminé. Cette communauté de bactéries est la principale responsable de la méthylation tant en milieu marin qu'en eau douce dans un environnement riche en soufre (Duran et al., 2008). Cependant, il a été reconnu que les bactéries réductrices de fer et les bactéries méthanogènes sont aussi capables de methyler le mercure dans des conditions anoxiques en absence de sulfate même si leurs contributions sont jugées insignifiantes (Hamelin et al., 2011; Bridou et al., 2011; Gilmour et al., 2011 ; Schaefer et al., 2011; Gilmour et al., 2013).

\section{Processus de dé-méthylation}

Parallèlement à la méthylation du mercure inorganique, le méthylmercure peut se dégrader au sein même du sédiment et de la colonne d'eau par des processus également biotiques et/ou abiotiques (Yu et al., 2012).. Ainsi dans les sédiments riches en sulfures, le mercure organique peut se décomposer chimiquement en sulfures mercuriques qui précipite (Duran et al., 2008)

Dans les systèmes sédimentaires, la déméthylation du mercure est principalement un procédé microbien et la plupart des bactéries méthylantes sont également capables de déméthyler le methylmercure (Bridou et al., 2011). Aussi, la dé-méthylation par voie oxydative est observée chez certaines bactéries sulfate-réductrices et méthanogènes. Il aboutit à la formation de dioxyde de carbone $\left(\mathrm{CO}_{2}\right)$ selon $\mathrm{Yu}$ et al., (2012). De plus, les bactéries réductrices du fer interviennent activement dans la déméthylation du mercure (Bridou et al., 2011 ; Yu et al., 2012). 


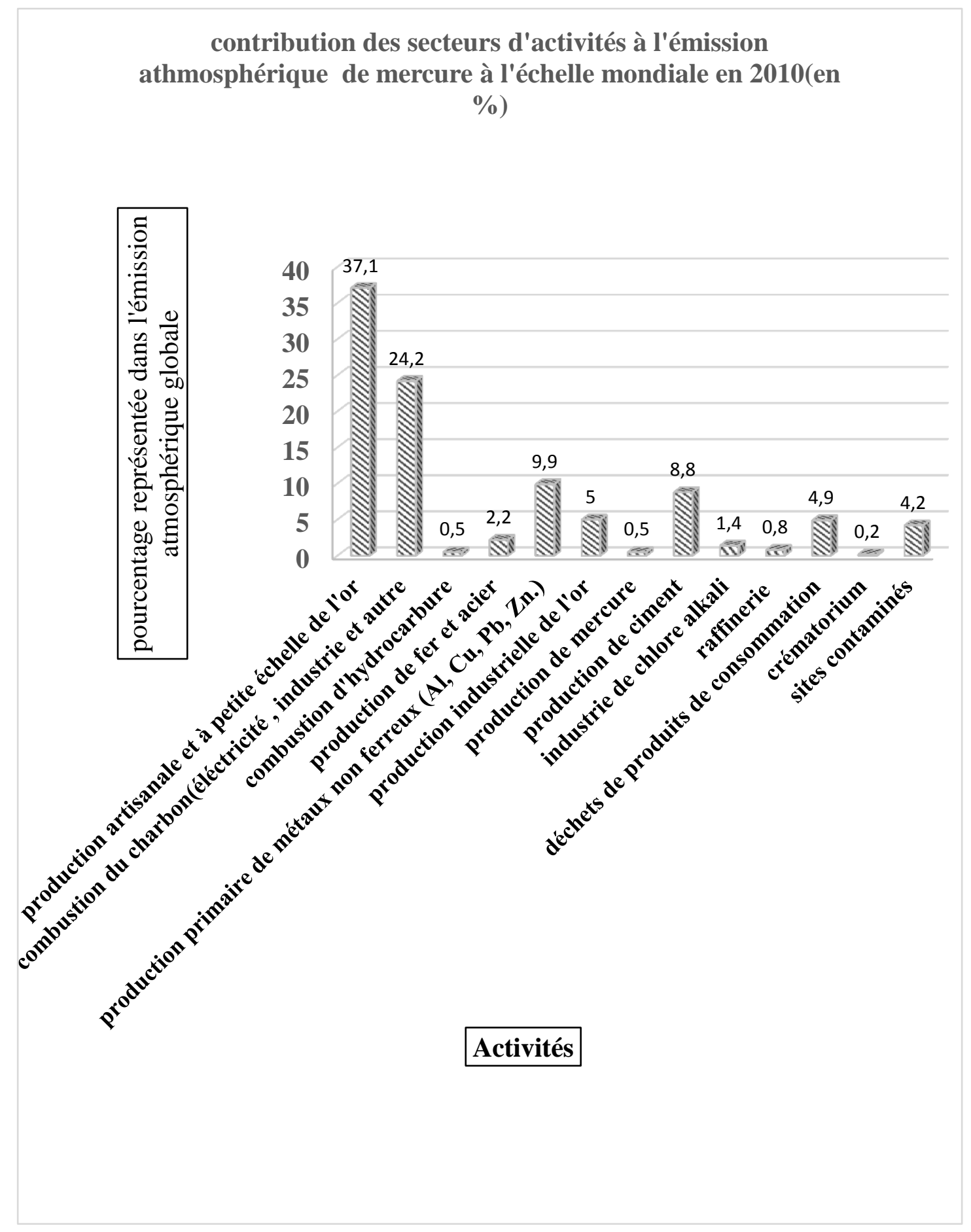

Figure 1: Contribution des secteurs d'activité à l'émission anthropique du mercure dans l'atmosphère (Réalisée sur la base des données de AMAP, 2013). 


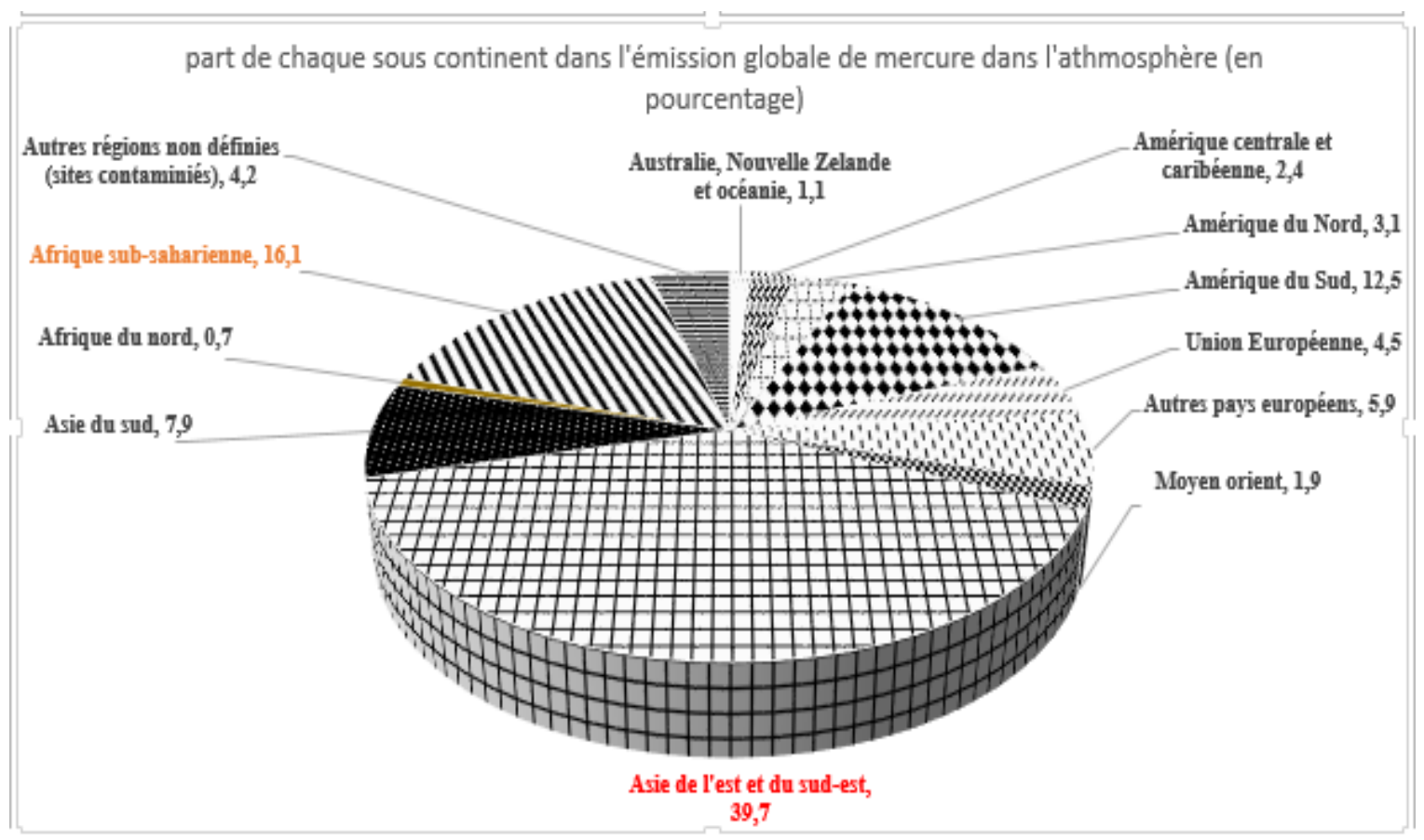

Figure 2 : Contribution des régions à l'émission anthropique du mercure dans l'atmosphère (Réalisé à partir des données de AMAP, 2013).

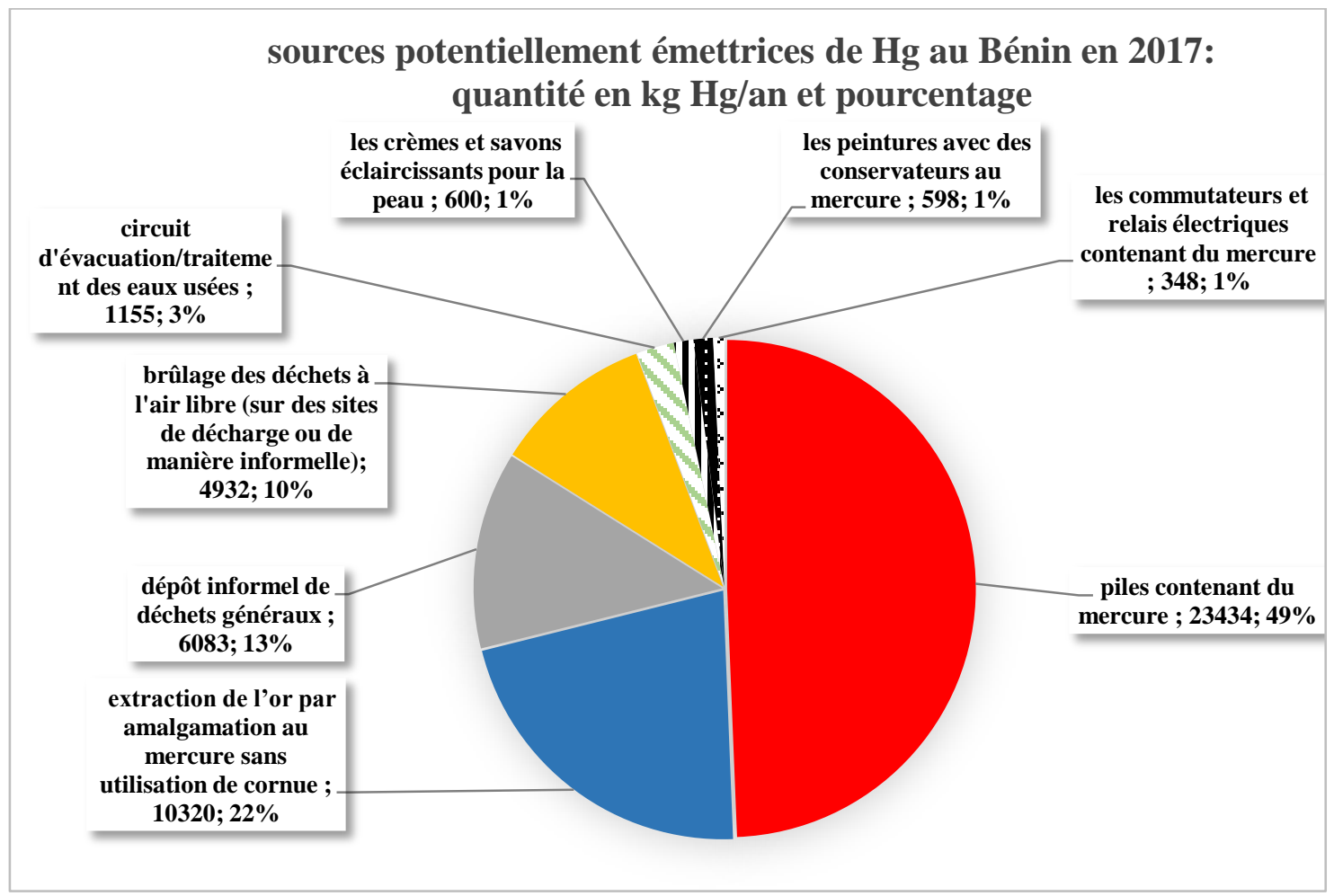

Figure 3 : Source d'apport de mercure au Bénin (graphique réalisé à partir des données du Rapport de l'inventaire initial du mercure au Bénin, 2017). 


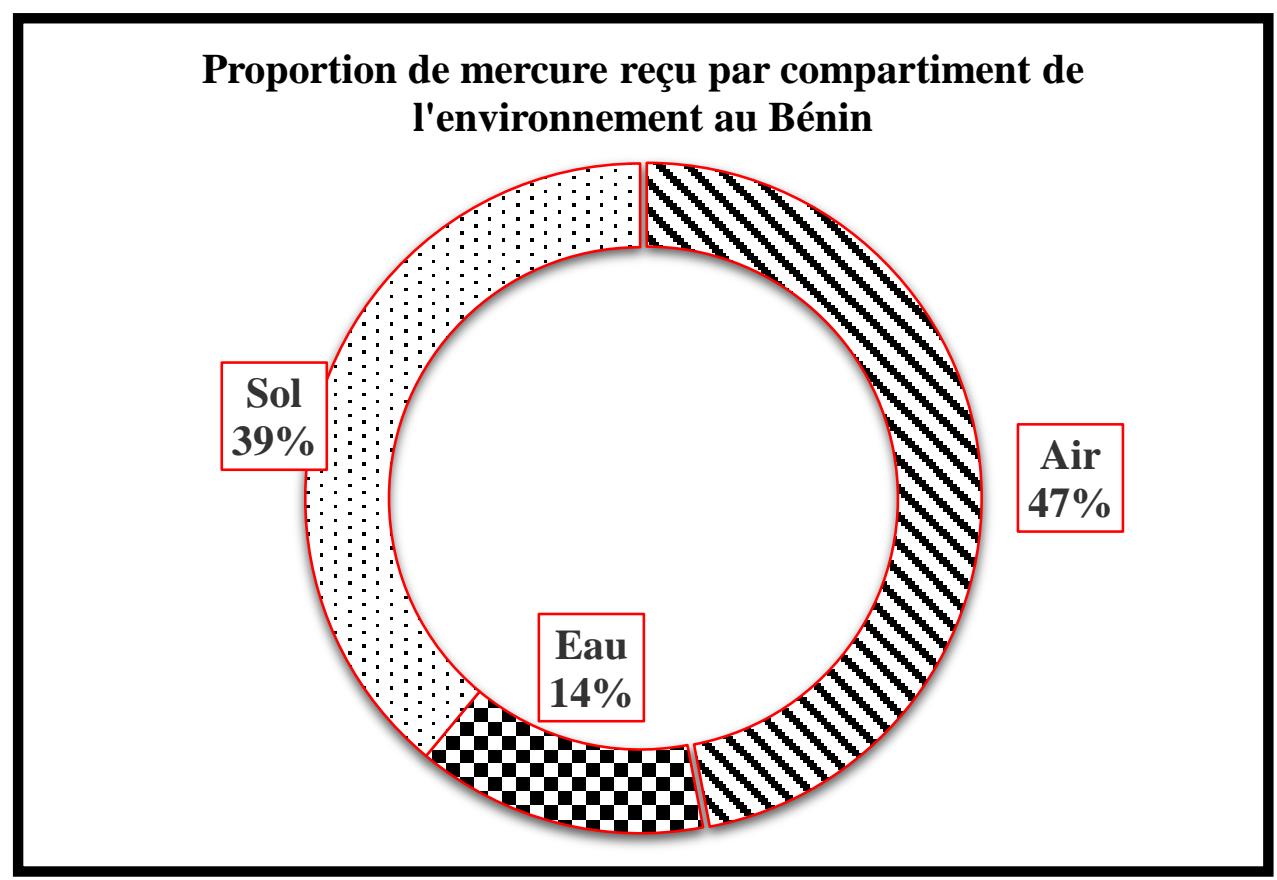

Figure 4: Proportion de mercure reçu par les compartiments de l'environnement au Bénin en 2017 (graphique réalisé à partir des données du rapport de l'inventaire initial du mercure, 2017).

Tableau 1: sources d'émission/rejet de mercure et quantités émises par compartiment de l'environnement au Bénin exprimée en tonnes de mercure par an (rapport d'évaluation initial 2017).

\begin{tabular}{llll}
\hline Compartiments de l'environnement & $\begin{array}{l}\text { Air (t de } \\
\text { Hg/an) }\end{array}$ & Eau (t de Hg/an) & Sol (t de Hg/an) \\
\hline Piles contenant du mercure & 5,86 & 5,86 \\
\hline Brûlage à l'air libre & 4,93 & & 2,89 \\
\hline Extraction de l'or & 4,14 & 3,3 & 4,87 \\
\hline $\begin{array}{l}\text { Dépôts informels de déchets } \\
\text { Crèmes et savons éclaircissants }\end{array}$ & 0,61 & & \\
contenant du mercure & 0,57 & 2,88 & 0,20 \\
\hline $\begin{array}{l}\text { Circuits d'évacuation des eaux usées } \\
\text { Cimetières }\end{array}$ & 1,04 & 0,13 \\
\hline $\begin{array}{l}\text { Commutateurs et relais électriques } \\
\text { contenant du mercure }\end{array}$ & & \\
\hline
\end{tabular}




\section{DISCUSSION}

Emissions de mercure: variation des estimations et régions les plus émettrices.

Si le dégazage de la croute terrestre et les éruptions volcaniques constituent les principales sources naturelles de mercure dans l'atmosphère (Nriagu et Becker, 2003), une bonne partie du mercure naturel ou anthropique, après s'être déposée, se volatilise

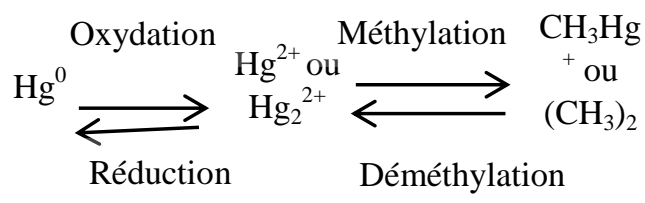

à nouveau puis se redistribue à nouveau dans les différents compartiments de l'environnement. Ainsi, le mercure une fois introduit dans l'environnement n'en sort plus. Il passe d'une espèce chimique à une autre comme le montre Poissant et al. (2002) à travers les principales voies de transformations chimiques et biologiques ciaprès ainsi que l'analyse du cycle biogéochimique du mercure dans l'environnement (Figure 5).

Les réémissions proviennent également de dépôts antérieurs liés aussi aux activités anthropiques (Gustin et al., 2008). De ce fait, l'estimation des émissions naturelles de mercure pourrait souffrir d'une grande imprécision. Ceci justifie la grande variation de l'estimation des émissions naturelles de mercure. En effet, pour certains auteurs les valeurs fluctuent entre 800 et 3000 tonnes par an et pour d'autres elles passent à 500 tonnes /an quand on exclut les émissions volcaniques (Nriagu et Becker, 2003) tandis que Pyle et Mather (2003) estime les émissions volcaniques seule à 700 tonnes par an. Quant aux émissions anthropiques de mercure, les auteurs s'accordent aisément sur les estimations qui varient très peu d'un auteur à un autre.

Une étude de la répartition géographique des émissions globales de mercure dont les valeurs sont estimées à environ 8900 tonnes par an (AMAP, 2013), montre que le développement économique serait l'une des raisons d'importante émission de mercure dans l'atmosphère. En effet, les régions ayant connues les plus fort taux d'accroissement de leur Produit intérieurs brut entre 1990 et 2010 comme l'Asie de l'Est et pacifique avec $259 \%$ d'accroissement du PIB ainsi que l'Afrique Sub-saharienne avec 340\% contribuent le plus aux émissions de mercure avec respectivement $39,7 \%$ et $16,1 \%$ des émissions mondiales de mercure dans l'atmosphère (Figue 2 et Tableau 3).

\section{Comportements du mercure en milieu aquatique et facteurs influençant sa méthylation}

Comme le montre le cycle bio géochimique du mercure (Figure 5) le milieu aquatique sert à la fois de source d'émission du mercure dans l'atmosphère et de réservoir de stockage. En effet, le mercure se volatilise des eaux de surface dans l'atmosphère par photo réduction du mercure divalent (Mason, 2009), se dépose dans les sédiments sous forme de $\mathrm{HgS}$ ou dans les organismes vivant sous forme de Methyl mercure (Figure 5).

En fonction du $\mathrm{pH}$ et de la salinité de l'eau, le mercure s'y présente majoritairement sous forme de complexes hydroxo ou chloro. De plus, les espèces de mercure inorganique $y$ sont souvent liées à la matière organique comme les acides humiques, fulviques ou carboxyliques, etc. (Zhang et Lindberg, 2001 ; Ravichandran, 2004). Dans les zones plus profondes des océans, le mercure divalent en se liant aux particules en suspension sédimente (Lindberg et al., 2007) et par un processus biotique ou abiotique, se transforme en méthylmercure, ou diméthylmercure. Au niveau des eaux douces, le mercure existe également sous forme de méthyl mercure. Mais, ici, en plus de la production in situ par les phénomènes abiotiques ou biologiques le méthylmercure provient également des eaux de ruissellement (Warner et al., 2003; Graydon et al., 2008). Au niveau des sédiments, le mercure peut avoir un temps de rétention très long en fonction de ses caractéristiques (Grigal, 2002 ; PNUE, 2002). Dans les sédiments anoxiques, le mercure est principalement présent sous forme de sulfure de mercure $\mathrm{HgS}$, préservé de la méthylation 
(Kampalath et al., 2013). Toutefois, comme au niveau de la colonne d'eau, les composés de mercure divalent peuvent être réduits en $\mathrm{Hg} 0$ via la lumière ou certaines substances humiques (Carpi et Lindberg, 1997) ou entrée dans un processus de méthylation/démethylation biotique ou abiotique (Hamelin et al., 2011; Yu et al., 2012). Le méthylmercure s'accumule dans la chaine trophique (Celo et al., 2006 ; Piraino et Taylor, 2009; Lehnherr et St Louis, 2009) et constitue une source de risques sanitaire pour l'homme par ingestion des produits halieutiques.

La méthylation du mercure est influencée par plusieurs composés tant organiques qu'inorganiques ainsi que les conditions physico chimique du milieu. Les plus importants sont le $\mathrm{pH}$, l'oxygène dissous, la forme chimique du mercure, la matière organique, les sulfure, le cycle de fer et du manganèse et la température.

Ullrich et al. (2001) constatent une augmentation de la production de méthylmercure au niveau de l'interface eausédiment oxique lorsque le $\mathrm{pH}$ diminue. Au contraire dans les sédiments anoxiques, la production nette de mercure organique décroît généralement avec la diminution $\mathrm{du} \mathrm{pH}$. Cependant, les conditions oxiques influencent faiblement à elles seules la méthylation dans les sédiments. C'est ce que précise Paranjape et Hall (2017) qui font remarquer que la méthylation se produit non seulement dans des conditions anoxiques, mais également en conditions oxiques car certaines bactéries méthylantes ne sont pas anaérobies.

Pour ce qui est du mercure, il est à noter que plus que le mercure total, c'est le mercure dissous principalement sous forme de molécule non chargée qui favorise la méthylation. En effet, la production microbienne du methylmercure nécessite un transport membranaire du mercure or les membranes bactériennes sont généralement plus perméables aux molécules non chargées (Ullrich et al., 2001). Benoit et al. (2001) ont montré qu'en présence de faible concentration de sulfure, la production de $\mathrm{MeHg}$ est proportionnelle à la concentration de mercure dissous et qu'elle décroit lorsque la concentration en sulfure augmente probablement du fait d'une diminution de la concentration de la forme neutre dissoute HgS0 biodisponible pour les bactéries. Ainsi la biodisponibilité en mercure serait en fait contrôlée par des complexes solubles et neutres du mercure (Ullrich et al., 2001). $\mathrm{HgCl}_{2}$ serait la principale forme chimique du mercure absorbée par les cellules dans les eaux oxiques (Thomassin et al., 2003). En revanche, pour les environnements anoxiques, les complexes dissous non chargés comme les sulfures mercuriques $\mathrm{HgS}$, les bisulfures $\mathrm{Hg}(\mathrm{HS})_{2}$ ou encore les polysulfures $\mathrm{HgSn}^{\circ}$ seraient plus assimilables par les bactéries (Benoit et al., 2001; Kampalath et al., 2013). Ainsi, en milieu oxique ou anoxique, c'est sous forme de complexe soluble neutre que le mercure est assimilable par les bactéries pour la synthèse du méthyl mercure.

Concernant la matière organique, son transfert de la colonne d'eau vers le compartiment sédimentaire a pour effet de stimuler l'activité bactérienne au niveau des sédiments de surface (Covelli et al., 2001). De plus, la dégradation de la matière organique au sein du sédiment entrâne la dissolution des oxydes et hydroxydes de fer et de manganèse et du mercure associé favorisant ainsi la méthylation (Cossa et Gobeil, 2000). Aussi, la présence de carbone organique dissous améliore de façon significative la solubilité de $\mathrm{HgS}$ (Ravichandran, 2004) évitant ainsi sa précipitation. Une eau riche en matière organique, favorise donc la méthylation du mercure (Warner et al., 2005; Ribeiro et al., 2008).

Concernant les sulfures, il est à retenir que dans les sédiments anaérobies, la présence de sulfate, augmente généralement la méthylation du mercure (Kampalath et al., 2013) en raison de son rôle d'accepteur d'électrons pour les bactéries sulfitoréductrices (Bigham et al., 2016) toutefois, une forte concentration en sulfure inhibe la formation de mercure organique au sein du sédiment par diminution de la disponibilité 
en mercure inorganique dissous qui se précipite sous forme de $\mathrm{HgS}$ (Duran et al., 2008). Ainsi, la biodisponibilité du mercure inorganique pour la méthylation dépend des espèces de sulfures de mercure formées. En effet, des espèces de sulfures de mercure non chargées (par exemple, Hg (SH) 2 (aq)) peuvent passer à travers les membranes des cellules bactériennes où elles peuvent être méthylées (Drott et al., 2007). La précipitation du sulfure ou les espèces chargées inhibent la méthylation (Kampalath et al., 2013) du fait de leur incapacité à traverser la barrière de la membrane bactérienne (Bigham et al., 2016).

Concernant le cycle du fer et du manganèse, Cossa et Gobeil (2000) ont montré que la concentration en méthylmercure est maximale juste en dessous de l'interface eau-sédiment à l'endroit même où les concentrations en mercure et fer dissous augmentent simultanément. Aussi, dans les couches superficielles oxiques des sédiments, l'association du mercure à la matière organique et aux oxydes et hydroxydes de fer et/ou de manganèse a pour effet de limiter les concentrations en mercure dissous dans les eaux interstitielles (Cossa et Gobeil, 2000). La présence de fer et de manganèse constituerait donc un facteur limitant pour la biodisponibilité de mercure en milieu superficiel oxique.

Pour ce qui est de la température, Thomassin et al. (2003) affirme que l'augmentation de la température favorise la méthylation du mercure du fait de son effet bénéfique sur l'activité bactérienne.

\section{Mécanisme d'action du méthyl mercure dans l'organisme humain}

La contamination au mercure touche toutes les populations et principalement les populations fortes consommatrices des produits halieutiques. Le méthylmercure a une grande solubilité dans les lipides et les solvants organiques, mais possède également une grande affinité pour les groupes thiols ($\mathrm{SH}$, particulièrement abondants dans les protéines. Ces propriétés lui confèrent une grande stabilité avec une période de demi-vie relativement longue: 70 jours dans le sang, et 270 jours dans le cerveau (Picot et Proust, 1998). La voie orale est la principale voie d'exposition au MeHg (95\% absorbé par l'organisme). Il est ingéré puis absorbé par l'estomac et le tractus gastro-intestinal. Le $\mathrm{MeHg}$ peut alors se lier aux groupements thiols de certains ligands comme la cystéine ou l'albumine. Le complexe MeHg-cystéine mime la méthionine, un acide aminé présent dans l'organisme, ce qui permet au $\mathrm{Hg}$ d'entrer dans les cellules et d'être distribué dans l'ensemble de l'organisme via le système sanguin. Il est ensuite excrété par la bile sous la forme d'un complexe MeHg-glutathion, redistribuée par le système sanguin ou déméthylé et éliminé dans les fèces (Margos et Clarkson, 2006). Le Hg peut également se lier aux molécules constituant la cellule vivante (acide nucléique, protéines, etc.) modifiant ainsi leur structure et/ou inhibant leurs activités biologiques. Le $\mathrm{MeHg}$ se fixe préférentiellement aux cellules nerveuses et provoque leur dégénérescence par des réactions d'oxydation. Par le biais de transporteurs, le $\mathrm{MeHg}$ peut également franchir la barrière hémato-encéphalique et s'accumuler dans le cerveau ou la barrière foeto-placentaire et inhiber l'activité des enzymes responsables de la croissance des neurones lors du développement du cerveau, provoquant ainsi des malformations et des retards dans le développement de l'enfant (Budtz-Jorgensen et al., 2004 ; Bensefa- Colas et al., 2011; Young-Seoub et al., 2012; Grandjeana et al., 2014). Ce mécanisme justifie pleinement les nombreux troubles neurologiques de l'intoxication au mercure dont la synthèse st présenté dans le tableau1 
Tableau 3: produits intérieurs bruts par région.

\begin{tabular}{|c|c|c|c|}
\hline Régions/Pays & $\begin{array}{l}\text { PIB en Billion de } \\
\text { dollars US (1990) }\end{array}$ & 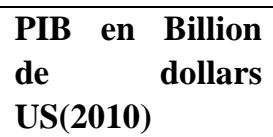 & $\begin{array}{l}\text { pourcentage } \\
\text { d'accroissement du PIB } \\
\text { entre } 1990 \text { et } 2010\end{array}$ \\
\hline Asie de l'Est et pacifique & 4,732 & 16,987 & $259 \%$ \\
\hline Afrique sub saharienne & 0,310 & 1,364 & $340 \%$ \\
\hline Amérique du Sud & 0,934 & 3,596 & $285 \%$ \\
\hline $\begin{array}{l}\text { Afrique du Nord et } \\
\text { Moyen Orient }\end{array}$ & 0,551 & 2,768 & $402 \%$ \\
\hline Asie du Sud & 0,402 & 2,040 & $407 \%$ \\
\hline Union Européenne & 7,573 & 16,987 & $124 \%$ \\
\hline Amérique du Nord & 6,575 & 16,584 & $152 \%$ \\
\hline
\end{tabular}

Source: https://donnees.banquemondiale.org/ consulté ce 22/01/2019.

\section{Transport atmosphérique

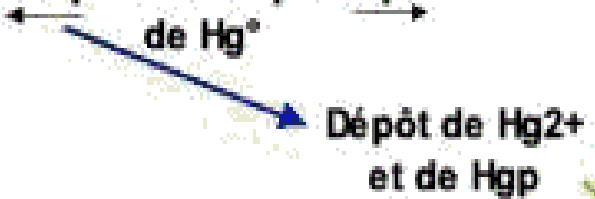

Atmosphère

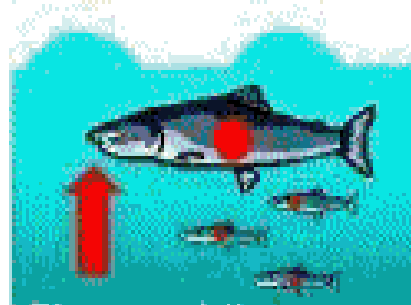

Bromecumbtation

\section{Eau} $\mathrm{MeHg}$

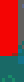

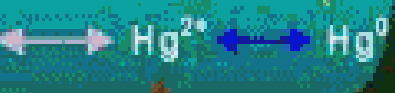

MeHg
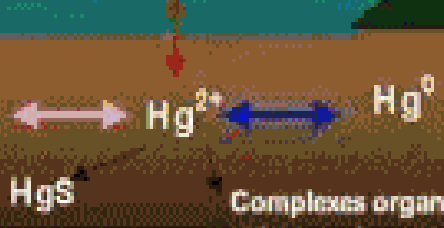

\section{Sédiment} Hgs

Complexes organiques et inorganiques

Evaporation

Figure 5: Cycle biogéochimique du mercure dans l'environnement source: https://www.canada.ca/fr/environnement-changement-climatique/services/polluants/mercureenvironnement/a-propos/biogeochimie.html\#cyclebiogeochimique. 


\section{Conclusion}

Le mercure est une substance toxique omniprésente dans tous les compartiments de l'environnement. Sa concentration est en augmentation du fait des activités anthropiques. La présente revue de littérature, un travail précurseur à celui de l'évaluation de la teneur du $\mathrm{Hg}$ dans les produits halieutiques des lacs et lagunes au sud du Bénin, a permis principalement d'appréhender l'ampleur de sa toxicité une fois qu'il est consommé sous différentes formes chimiques par voie digestive, respiratoire ou cutanée. Les recherches ont indiquées qu'au Bénin, le mercure en milieu aquatique constitue un danger potentiel tant pour la santé humaine que la vie aquatique principalement au niveau du Mekrou et de la lagune de Porto-Novo.

\section{CONFLIT D'INTERETS}

Nous auteurs de cet article déclarons n'avoir aucun conflit d'intérêt.

\section{CONTRIBUTIONS DES AUTEURS}

HWD a rédigé cet article avec la contribution des autres auteurs. C'est le premier auteur de cet article. NBNA: A enrichi cet article par la mise à disposition de documents et articles ainsi que la lecture de l'article rédigé. JGA a supervisé le travail, a amélioré la rédaction et a fait l'assurance qualité du document. AVOA a contribué à cet article par la mise à disposition des documents de l'inventaire national du mercure au Bénin qu'il a réalisé au Bénin en 2017. MPA a contribué à l'amélioration de l'article par ses conseils et a contribué à l'assurance qualité de l'article.

\section{REMERCIEMENTS}

Nous remercions le Point Focal National de la Convention de Minamata sur le Mercure, Mme Sandra GONOU pour sa disponibilité et les informations fournies.

\section{REFERENCES}

Adam S, Edorh P, Totin H, Koumolou L, Amoussou E, Aklikokou K, Boko M. 2010. Pesticides et métaux lourds dans l'eau de boisson, les sols et les sédiments de la ceinture cotonnière de Gogounou,
Kandi et Banikoara (Bénin). Int. J. Biol. Chem. Sci., 4(4) : 1141-1150.

Adamou MR, Pazou EYA, Deguenon AY. 2019. Contamination des escargots prélevés dans la vallée de l'Ouémé au Bénin par des métaux lourds et évaluation des risques sanitaires. International Journal of Biological and Chemical Sciences, 13(1): 126-144. DOI: https://dx.doi.org/10.4314/ijbcs.v13i1.11

Aïna MP, Degila H, Chikou A, Adjahatode F, Matejka G. 2012. Risk of intoxication by heavy metals (pb, cd, cu, hg) connected to the consumption of some halieutic species in lake nokoue: case of the penaeus shrimps and the sarotherodon melanotheron. British Journal of Science, 5(1): 104-118.

AMAP/UNEP [Arctic Monitoring and Assessment Programme/United Nations Environment Programme]. 2013. Technical Background. Report for the Global Mercury Assessment UNEP Chemicals Branch, Geneva, Switzerland, 36p. https://oaarchive.arcticcouncil.org/handle/11374/732

Bensefa-Colas L, Andujar P, Alexis DL. 2011. Intoxication par le mercure. $L a$ Revue De Médecine Interne, 32 (7): 416-424. DOI: https://doi.org/10.1016/j.revmed. 2009.08.024

Benoit JM, Gilmour CC, Mason RP. 2001. Aspects of bioavailability of mercury for methylation in pure cultures of Desulfobulbus propionicus (1pr3). Applied and Environmental Microbiology, 67(1): 51-58. DOI: http://dx.doi.org/ 10.1128/AEM.67.1.5158.2001

Bose-O'Reilly S, Schierl R, Nowak D, Siebert U, William JF, Owi FT, Ir YI. 2016. A preliminary study on health effects in villagers exposed to mercury in a smallscale artisanal gold mining area in Indonesia. Environmental Research, 149, 274-281.

DOI: http://dx.doi.org/10.1016/j.envres.2016.0 4.007 .

Bridou R, Monperrus M, Rodriguez-Gonzalez P, Guyoneaud R, Amouroux D. 2011. 
Simultaneous Determination of Mercury Methylation and Demethylation Capacities of Various Sulfate-reducing Bacteria Using Species-specific Isotopic Tracers. Environmental Toxicology and Chemistry, 30: 1-8. DOI: http://dx.doi.org/10.1002/etc.395

Budtz-Jorgensen E, Grandjean P, Jorgensen PJ, Weihe P, Keiding N. 2004. Association between mercury concentrations in blood and hair in methylmercury-exposed subjects at different ages. Environ Res., 95: 385-93. DOI: https://doi.org/10.1016/j.envres.2 003.11.001

Carpi A, Steven E, Lindberg EM, Prestbo, Nicolas SB. 1997. Methyl Mercury Contamination and Emission to the Atmosphere from Soil Amended with Municipal Sewage Sludge. Journal of Environmental Quality, 26(6): 16501655.

DOI:10.2134/jeq1997.004724250026000 $60027 \mathrm{x}$

Celo V, Lean DRS, Scott SL. 2006. Abiotic methylation of mercury in the aquaticenvironment. Science of The Total Environment, 368(1): 126-137. DOI: https://doi.org/10.1016/j.scitotenv.2005. 09.043

Choe KY, Gill GA, Ronald D, Lehman SH. 2004. Sediment-water exchange of total mercury and monomethyl mercury in the San Francisco Bay-Delta. Limnol. Oceanogr., 49(5): 1512-1527. DOI: https://doi.org/10.4319/lo.2004.49.5.151 2

Chouti W, MAMA D, Alapini F. 2010. Etude des variations spatio-temporelles de la pollution des eaux de la lagune de PortoNovo (sud Bénin). Int. J. Biol. Chem. Sci., 4(4) : 1017-1029

Chouti W, Mama D, Alassane Ak, Changotade O, Alapini F, Boukari M, Aminou T, Afouda A. 2011. Caractérisation physicochimique de la lagune de Porto-Novo (sud Bénin) et mise en relief de la pollution par le mercure, le cuivre et le zinc. Journal of Applied Biosciences, 43: 28822890.
Cossa D, Gobeil C. 2000. Mercury speciation in the Lower St. Lawrence estuary. Canadian Journal of Fisheries and Aquatic Sciences, 57(1): 138-147. DOI: https://doi.org/10.1139/cjfas-57-S1-138

Crespo-Lopez ME, Macedoa GL, Pereirab SI, Arrifanoa GP, Picanco-Diniz DL, do Nascimento JL. 2009. Mercury and human genotoxicity: critical considerations and possible molecular mechanisms. Pharmacological Research, 60: 212-220. DOI: https://doi.org/10.1016/j.phrs.2009.02.01 1.

Covelli S, Faganeli J, Horvat M, Brambati A. 2001. Mercury contamination of coastal sediments as the result of long term cinnabar mining activity (gulf of Trieste, northern Adriatic sea). Applied Geochemistry, 16: 541-558. DOI: https://doi.org/10.1016/S08832927(00)00042-1

Diez S. 2009. Human health effects of methylmercury exposure. Rev. Environ. Contam. Toxicol., 198:111-32. DOI: https://doi.org/10.1007/978-0-38709647-6_3.

Direction Généra de l'Environnement et du Climat. 2018. Evaluation initiale du mercure au Bénin. Rapport de la Direction Généra de l'Environnement et du Climat, 182P.

Drake PL, Rojas M, Reh CM, Mueller CA, Jenkins FM. 2001. Occupational exposure to airborne mercury during gold mining operations near El Callao, Venezuela. Int. Arch. Occup. Environ. Health., 74(3): 206-212. DOI: https://doi.org/10.1007/s004200000206

Driscoll CT, Han YJ, Chen CY, Evers DC, Fallon Lambert K, Holsen TM, Neil C, Kamman NC, Munson RK. 2007. Mercury Contamination in Forest and Freshwater Ecosystems in the Northeastern United States. Bio Science, 57(1): 17-28. DOI: https://doi.org/ 10.1641/b57010.

Drott A, Lambertsson L, Bjorn E, Skyllberg U. 2007. Importance of dissolved neutral mercury sulfides for methyl mercury production in contaminated sediments. Environ. Sci. Technol., 41: 2270-2276. 
DOI:

https://dx.doi.org/10.1021/es061724z

Duran R, Ranchou-Peyruse M, Menuet V, Monperrus M, Bareille G, Goñi MS, Salvado JC, Amouroux D, Guyoneaud R, Donard OFX, Caumette P. 2008. Mercury methylation by a microbial community from sediments of the Adour Estuary (Bay of Biscay, France). Environmental Pollution, 156(3): 951958.

DOI: https://doi.org/10.1016/j.envpol.2008.05. 007

Edorh PA, Agonkpahoun E, Gnandi K, Guedenon P, Koumolou L, Amoussou C, Ayedoun AM, Boko M, Gbeassor M, Rihn BH, Creppy EE. 2009. An assessment of the contamination of Achatina achatina by toxic metals in Okpara village (Benin). Int. J. Biol. Chem. Sci., 3(6): 1428-1436. DOI: http://dx.doi.org/10.4314/ijbcs.v3i6.5354

Fitzgerald WF, Lamborg CH, Hammerschmidt CR. 2007. Marine biogeochemical cycling of mercury. Chem. Rev., 10: 641-662. DOI: https://doi.org/10.1021/cr050353m

Bigham GN, Murray KJ, Masue-Slowey Y, Henry AE. 2016. Biogeochemical Controls on Methylmercury in Soils and Sediments: Implications for Site Management. Integr Environ Assess Manag., 13: 249-263. DOI: https://doi.org/10.1002/ieam.1822

Gardner RM, Nyland JF, Silbergeld EK. 2010. Differential immunotoxic effects of inorganic and organic mercury species in vitro. Toxicol. Lett., 198 (2):182-190. DOI:

https://doi.org/10.1016/j.toxlet.2010.06.0 15

Gibb H, O’Leary KG. 2014. Mercury exposure and health impacts among individuals in the artisanal and smallscale gold mining community: a comprehensive review. Environ. Health Perspect., 122: 667-672. DOI: http://dx.doi.org/10.1289/ehp.1307864.

Gilmour CC, Elias DA, Kucken AM, Brown SD, Palumbo AV, Schadt CW, Wall JD. 2011. Sulfate-Reducing Bacterium Desulfovibrio desulfuricans ND132 as a
Model for Understanding Bacterial Mercury Methylation. Applied and Environmental Microbiology, 77(12): 3938-3951.

DOI: http://dx.doi.org/10.1128/AEM.0299310

Gilmour CC, Podar M, Bullock AL, Graham AM, Brown SD, Somenahally AC, Johs A, Hurt RA, JR, Bailey KL, Elias DA. 2013. Mercury Methylation by Novel Microorganisms from New Environments. Environ. Sci. Technol., 47: $\quad 11810-11820 . \quad$ DOI: http://dx.doi.org/10.1021/es403075t

Grandjeana P, Weihec P, Frodi D, Anna L, Choib, BudtzJørgensen E. 2014. Neurotoxicity from prenatal and postnatal exposure to methylmercury. Neurotoxicol. Teratol., 43: 39-44. DOI: http://dx.doi.org/10.1016/j.ntt.2014.03.0 04.

Graydon JA, St Louis VL, Hintelmann H, Lindberg SE, Sandilands KA, Rudd JW, Kelly CA, Hall BD, Mowat LD. 2008. Long-term wet and dry deposition of total and methyl mercury in the remote boreal ecoregion of Canada. Environ. Sci. Technol., 42(22): 8345-8351. DOI: http://dx.doi.org/10.1021/es801056j

Grigal DF. 2002. Inputs and outputs of mercury from terrestrial watersheds: a review. Environmental research, 10: 139. DOI: https://doi.org/10.1139/a01-013

Gustin MS, Lindberg S, Weisberg P. 2008. An update on the natural sources and sinks of atmospheric mercury. Applied Geochemistry, 23(3): 482-49. DOI: https://doi.org/10.1016/j.apgeochem.200 7.12.010

Hamelin S, Amyot M, Barkay T, Wang Y, Planas D. 2011. Methanogens: Principal Methylators of Mercury in Lake Periphyton. Environmental Science \& Technology, 45(18): 7693-7700. DOI: https://doi.org/10.1021/es2010072

Harari R, Harari F, Gerhardsson L, Lundh T, Skerfving S, Strömberg, Broberg K. 2012. Exposure and toxic effects of elemental mercury in gold-mining activities in Ecuador. Toxicology Letters, 213: 75-82.

DOI: 
https://doi.org/10.1016/j.toxlet.2011.09.0 06

Holmes CD, Jacob DJ, Corbitt ES, Mao J, Yang X, Talbot R, Slemr F. 2010. Global atmospheric model for mercury including oxidation bybromine atoms. Atmos. Chem. Phys., 10: 12037-12057. DOI: https://doi.org/10.5194/acp-1012037-2010

Hounkpatin ASY, Edorh AP, Salifou S, Gnandi K, Koumolou L, Agbandji L, Aissi KA, Gouissi M, Boko M. 2012. Assessment of exposure risk to lead and cadmium via fish consumption in the lacusrian village of Ganvié in Benin republic. Journal of Environmental Chemistry and Ecotoxicology, 4(1): 110.

DOI:

https://doi.org/10.5897/JECE11.063

INERIS. 2006. Mercure et ses dérives. Fiche de données toxicologiques et environnementales des substances dangereuses $\mathrm{n}^{\circ}$ DRC-00-2559099DF389, version $\mathrm{n}^{\circ} 3$. http://www.ineris.fr/substances/fr/substa nce/getDocument/2802

Kampalath RA, Lin C, Jay JA. 2013. Influences of zero-valent sulfur on mercurymethylation in bacterial cocultures. Water Air Soil Pollut., 224: $1-14$. DOI: https://doi.org/10.1007/s11270-0121399-7

Katemo MB, Colinet G, Andre L, Chocha Manda A, Marquet JP, Micha JC. 2010. Evaluation de la contamination de la chaîne trophique par les éléments traces ( $\mathrm{Cu}, \mathrm{Co}, \mathrm{Zn}, \mathrm{Pb}, \mathrm{Cd}, \mathrm{U}, \mathrm{V}$ et As) dans le bassin de la Lufira supérieure (Katanga/RD Congo). Tropicultura, 28(4): 246-252.

Lehnherr I, St Louis VL. 2009. Importance of ultraviolet radiation in the photodemethylation of methylmercury in freshwater ecosystems. Environmental Science \& Technology, 43(15): 56925698. DOI: https://doi.org/10.1021/es9002923

Lindberg $S$, Bullock R, Ebinghaus R, Engstrom D, Feng XB, Fitzgerald W, Pirrone N, Prestbo E, Seigneur C. 2007. A synthesis of progress and uncertainties in attributing the sources of mercury in deposition. AMBIO. A. J. Human Environment, 36(1): 19-32. DOI: https://doi.org/10.1579/0044-7447 (2007)36[19: ASOPAU] 2.0.CO; 2

Magos L, Clarkson TW. 2006. Overview of the clinical toxicity of mercury. Ann. Clin. Biochem., 43: 257-268 DOI: https://doi.org/10.1258/00045630677769 5654

Marusczak N. 2010. Etude du transfert du mercure et du méthylmercure dans les écosystèmes lacustres alpins. Thèse de doctorat, Université de Grenoble, France, $207 \mathrm{P}$.

Mason R, Pirrone N. 2009. Mercury emissions from natural processes and their importance in the global mercury cycle. In Mercury Fate and Transport in The Global Atmosphere, Mason R, Pirrone N (eds). Springer Nature: Switzerland AG. DOI: https://doi.org/10.1007/978-0-38793958-2_7

Mason RP, Sheu GR, 2002. Rôle of the ocean in the global mercury cycle. Global Biogeochemical Cycles, 16(4): 1-40. DOI: https://doi.org/doi:10.1029/2001GB0014 40.

Nriagu J, Becker C. 2003. Volcanic emissions of mercury to the atmosphere: global and regional inventories. Science of the Total Environment, 304: 3-12. DOI: https://doi.org/10.1016/S00489697(02)00552-1

Nyland JF, Fillion M, Barbosa FJ, Shirley DL, Chine C, Lemire $\mathrm{M}$, Mergler D, Silbergeld EK. 2011. Biomarkers of methylmercury exposure immunotoxicity among fish consumers in Amazonian Brazil. Environ. Health Perspect., 119(12): 1733-1748. DOI: https://doi.org/10.1289/ehp.1103741

OMS. 2003. Elemental mercury and inorganic mercury compounds: human health aspects. OMS. http://www.who.int/ipcs/publications/cic ad/en/cicad50.pdf.

Pacyna EG, Pacyna JM, Sundseth K, Munthe J, Kindbom K, Wilson S, Steenhuisen F, Maxson P. 2010. Global emission of mercury to the atmosphere from 
anthropogenic sources in 2005 and projections to 2020. Atmospheric Environment, 44: 2487-2499. DOI: https://doi.org/10.1016/j.atmosenv.2009. 06.009

Paranjape AR, Hall BD. 2017. Recent advances in the study of mercury methylation in aquatic systems. FACETS, 2: 85-119. DOI: https://doi.org/10.1139/facets-2016-0027

Morcillo P, Esteban MA, Cuesta A. 2017. Mercury and its toxic effects on fish AIMS. Environmental Science, 4(3): 386-402.

DOI: https://doi.org/10.3934/environsci.2017. 3.386

Picot A, Proust N. 1998. Le mercure et ses composés. De la spéciation à la toxicité. Actualite Chimique, 4: 16-24.

Piraino MN, Taylor DL. 2009. Bioaccumulation and trophic transfer of mercury in striped bass and tautog from the Narragansett Bay (Rhode Island, USA). Marine Environmental Research, 67(3): 117-128. DOI: https://doi.org/10.1016/j.marenvres.2008 .12 .006

PNUE. 2002. Evaluation mondiale du mercure. PNUE - Substances chimiques Genève, Suisse. Rapport d'étude du PNUE,

306p. https://wedocs.unep.org/bitstream/handle /20.500.11822/11721/final-

assessmentreport-Frevised.pdf?sequence $=1 \&$ isAllowed $=\mathrm{y}$

Poissant L, Dommergue A, Ferrari CP. 2002. Mercury as a global polluant. Journal de Physique IV, 12(10): 143-160. DOI: https://doi.org/10.1051/jp4:20020457

Pyle DM, Mather TA. 2003. The importance of volcanic emissions for the global atmospheric mercury cycle. Atmospheric Environment, 37(36): 51155124. DOI: https://doi.org/10.1016/j.atmosenv.2003. 07.011

Ranchou-Peyruse M, Monperrus M, Bridou R, Duran R, Amouroux D, Salvado JC, Guyoneaud R. 2009. Overview of Mercury Methylation Capacities among Anaerobic Bacteria Including Representatives of the Sulphate-
Reducers: Implications for Environmental Studies. Geomicrobiology Journal, 26: 1-8. DOI: https://doi.org/ 10.1080/01490450802599227

Ravichandran M. 2004. Interactions between mercury and dissolved organic matter. A Review. Chemosphere, 55: 319-331. DOI: https://doi.org/10.1016/j.chemosphere.20 03.11.011

Ribeiro Guevara S, Queimaliños CP, Diéguez Mdel C, Arribére M. 2008. Methylmercury production in the water column of an ultraoligotrophic lake of Northern Patagonia, Argentina. Chemosphere, 72(4): 578-85. DOI: https://doi.org/10.1016/j.chemosphere.20 08.03.011

Schaefer JK, Rocks SS, Zheng W, Liang L, Gu B, Morel FM. 2011. Active transport, substrate specificity, and methylation of $\mathrm{Hg}$ (II) in anaerobic bacteria. Proc. Natl. Acad. Sci. USA., 108(21): 8714-8719. https://doi.org/10.1073/pnas.1105781108

Selin NE, Daniel JJ, Robert M, Yantosca SS, Lyatt J, Elsie MS. 2008. Global 3-D land-ocean-atmosphere model for mercury: Present-day vs. preindustrial cycles and anthropogenic enrichment factors for deposition. Global Biogeochemical Cycles, 22(GB2011): 113.

DOI: https://doi.org/10.1029/2007GB003040

Robinson AS, Forbes MR, Hebert CE, Scheuhammer AM. 2011. Evidence for Sex Differences in Mercury Dynamics in Double-Crested Cormorants. Environ. Sci. Technol., 45(4): 1213-1218. DOI: https://doi.org/10.1021/es1021872

Stein ED, Cohen Y, Winer AM. 2009. Environmental Distribution and Transformation of Mercury Compounds. Critical reviews Environmental Science and Technology, 26(1): 1-43. DOI: https://doi.org/10.1080/10643389609388 485

Stella E, Anja E, Leonhard T, Natalia M, Tatyana P, Margit S. 2017. A 320 Year Ice-Core Record of Atmospheric $\mathrm{Hg}$ Pollution in the Altai, Central Asia. Environ. Sci. Technol., 51(20): 11597- 
11606.

DOI:

https://doi.org/10.1021/acs.est.7b03140.

Thomassin JF, Touzé S, Baranger PH. 2003. le mercure et ses composes: comportement dans les sols, les eaux et les boues de sediments. BRGM/RP, $121 \mathrm{P}$.

Tomicic C1, Vernez D, Belem T, Berode M. 2011. Human mercury exposure associated with small-scale gold mining in Burkina Faso. Int Arch Occup Environ Health, 84(5): 539-546. DOI: 10.1007/s00420-011-0615-x. Epub 2011 Jan 29.

Ullrich SM, Tanton TW, Abdrashitova SA. 2001. Mercury in aquatic environment: a review of factors affecting methylation. Critical Reviews in Environmental Science and Technology, 31(3): 241-293. DOI:

https://doi.org/10.1080/20016491089226

UNEP Global Mercury Assessment. 2013. Sources, Emissions, Releases and Environmental Transport. UNEP Chemicals Branch, Geneva, Switzerland, $44 \mathrm{p}$.

wedocs.unep.org/handle/20.500.11822/7 984

UNEP Chemicals Branch. 2008. The Global Atmospheric Mercury Assessment: Sources, Emission and transport. UNEPChemicals, Geneva. 44p.

Warner KA, Roden, EE, Bonzongo, JC. 2003. Microbial mercury transformation in anoxic freshwater sediments under ironreducing and other electron-accepting conditions. Environ. Sci. Technol., 37: 2159-2165.

DOI: https://doi.org/10.1021/es0262939

Warner KA, Bonzongo JC, Roden EE, Ward GM, Green AC, Chaubey I, Lyons WB, Arrington DA. 2005. Effect of watershed parameters on mercury distribution in different environmental compartments in the Mobile Alabama River Basin, USA. Sci. Total Environ., 347 : 187-207. DOI: https://doi.org/10.1016/j.scitotenv.2004.1 2.011 .
Yard EE, Horton J, Schier JG, Caldwell K, Sanchez C, Lewis L, Gastañaga C. 2012. Mercury exposure among artisanal gold miners in Madre de Dios, Peru: A Crosssectional study. J. Med. Toxicol., 8(4): 441-448.

DOI:

https://doi.org/10.1007/s13181-012-

0252-0

Young-Seoub H, Yu-Mi K, Kyung-Eun L. 2012. Methylmercury Exposure and Health Effects. J. Prev. Med. Public Health, 45(6): 353-363. DOI: http://dx.doi.org/10.3961/jpmph.2012.45 .6 .353

Youssao A, Soclo HH, Bonou C, Fayomi B. 2011. Evaluation de la bioaccumulation du plomb dans les espèces animales marines et identification des sources de contamination métallique par une analyse multiélémentaire en métaux ( $\mathrm{Al}$, $\mathrm{Cd}, \mathrm{Cr}, \mathrm{Cu}, \mathrm{Pb}$ ) dans les eaux côtières du Bénin. Int. J. Biol. Chem. Sci., 5(1): $188195 . \quad$ DOI: http://dx.doi.org/10.4314/ijbcs.v5i1.6809 7

Youssao AAK, Daouda M, Alassane MAK, Mama D, Youssao IAK. 2018. Sources and Distribution of Mercury Residues in Environmental and Food Matrices of the Mekrou River Watershed in Kèrou, Kouandé, Péhunco in Republic of Benin. American Journal of Applied Chemistry, 6(2): 57-63. DOI: https://doi.org/ 10.11648/j.ajac.20180602.14

Yu RQ, Flanders JR, Mack EE, Turner R, Bilal Mirza M, Barkay T. 2012. Contribution of coexisting sulfate and iron reducing bacteria to methylmercury production in freshwater river sediments. Environ. Sci. Technol., 46: 2684-2691. DOI: http://dx.doi.org/10.1021/es2033718

Zhang H, Lindberg S. 2001. Sunlight and Iron (III)-Induced Photochemical Production of Dissolved Gaseous Mercury in Freshwater. Environmental Science \& Technology, 25(5): 928-935. DOI: https://doi.org/10.1021/es001521p. 